\title{
ANGER AND ASSOCIATED EXPERIENCES OF SADNESS, FEAR, VALENCE, AROUSAL, AND DOMINANCE EVOKED BY VISUAL SCENES ${ }^{1,2}$
}

\author{
JOSÉ JULIÁN JAVELA \\ Institute for Biofunctional Studies \\ Department of Psycbobiology \\ Universidad Complutense de Madrid
}

\author{
ROBERTO E. MERCADILLO \\ Institute of Neurobiology \\ Universidad Nacional Autónoma de México
}

\section{J. MARTÍN RAMÍREZ}

Institute for Biofunctional Studies, Department of Psychobiology

Universidad Complutense de Madrid

Kennedy School of Government, Harvard University

\begin{abstract}
Summary.-Anger is a basic emotion experienced in several aversive situations. In this study, the relation between Anger, Fear, and Sadness, as well as the dimensions of Valence, Arousal, and Dominance, were examined. It was hypothesized that pictures showing an Intention to Harm would evoke not only Anger, but also Fear and Sadness, and that this would be correlated with low Valence, high Arousal, and high Dominance. To this end, 45 healthy volunteers (25 women and 20 men; $M$ age $=27.2$ yr., $S D=9.5$ ) recorded appraisals for each of these emotional experience while viewing 120 pictures selected from the International Affective Picture System. Data were analyzed with a linear mixed model and a hierarchical classes approach to identify differences and correlations between emotional categories. Results indicate that those pictures showing Intention to Harm generate higher Anger and Fear, while pictures representing Frustration of Goals leads to higher Sadness. In addition, high Anger, Fear, and Sadness are associated with low Valence, moderate Arousal, and high Dominance. These findings suggest that the experience of Anger requires the inference of the other's mental and physical state and need the regulation of cognitive and affective systems acting together. The study of this emotion should consider both categorical and dimensional approaches in order to define its coherent features.
\end{abstract}

Darwin's thesis about the innate component of emotional expressions $(1872 / 1988)$ has served to sustain the concept of emotion inside evolutionary mechanisms that aid an organism to alternate motivational systems and regulate environmental cues (Plutchik, 1980). Considering emotional expressions

\footnotetext{
'Addresss correspondence to Dr. J. Martín Ramírez, P.O. Box 2, 28792 Miraflores (Madrid), Spain or e-mail (mramirez@med.ucm.es).

'This study was supported by the program Alban, the European Union program of high level scholarship for Latin America, scholarship No. E04D033752CO, by the grant PR41/06-14942 to the Aggression Research Group of the Universidad Complutense de Madrid, and by CONACYT (194470). We gratefully acknowledge the contribution of Iwin Leenen with respect to the statistical analyses and his kind advice in the preparation of the manuscript. We thank Peter J. Lang, Margaret M. Bradley, and Bruce N. Cuthbert for their generous donation of the database and pictures of the International Affective Picture System (IAPS) and those volunteers who cordially participated in this study. Thanks to Magda Giordano for assistance with revisions.
}

DOI 10.2466/PR0.103.3.663-681 
and motivational systems, there are two forms in the modeling and classification of human emotions: a discrete model, focused on the recognition of primary emotions by analyzing universal patterns of expressions, and a dimensional model, referring to the identification of the fundamental variables or dimensions of the affective system.

According to the discrete model, the main elements of a primary or basic emotion comprise distinctive universal physiological signals and elicitors, for example, distinctive appearance and distinctive thoughts, memories, images, and subjective experience (Izard, 1992; Ekman, 1999). The number of basic emotions differs among researchers, but there is a consensus regarding six emotions: anger, fear, sadness, happiness, disgust, and surprise (Ortony \& Turner, 1990).

The dimensional model generally includes only two bipolar variables: hedonic valence (pleasant-appetitive motivation and unpleasant-defensive motivation) and arousal (motivational activation, from calm to excitation). This method based on appraisals is commonly tested by asking people who have experienced an emotion to report particular dimensions and concepts; for example, people rate the pleasure or displeasure associated with their experience when viewing a picture (Cuthbert, Bradley, \& Lang, 1996; Eisenberg, Sadovsky, \& Spinrad, 2005; Mikels, Fredrickson, Larkin, Lindberg, Maglio, \& Reuter-Lorenz, 2005).

These measures allow categorization of stimuli as elicitors of negative and positive emotions (Avero \& Calvo, 2006) and also permit identification of the physiological and motor aspects of emotions. For instance, negative valence and high arousal are associated with increased facial electromyographic activity (Bradley, Codispoti, Cuthbert, \& Lang, 2001; Bradley, Codispoti, Sabatinelli, \& Lang, 2001), and the startle reflex is inhibited by viewing positive pictures and increases when viewing negative pictures (Cuthbert, et al., 1996).

Some authors have combined the discrete and dimensional models with the aim of identifying the components and expressions of single emotions and clarifying whether dimensions are shared when two or more emotions are experienced. Researchers have reported that valence and arousal associated with viewing facial expressions of basic emotions differ in intensity according to the expression observed (Takehara \& Suzuki, 2001). The three negative emotions of disgust, sadness, and fear evoke different valence and arousal appraisals (Bradley, Codispoti, Cuthbert, \& Lang, 2001; Bradley, Codispoti, Sabatinelli, \& Lang, 2001), but positive emotions like happiness and satisfaction evoke similar intensities of both valence and arousal (Díaz \& Flores, 2001).

One primary emotion is anger, defined as a psychobiological state associated with a subjective experience that over time and across situations usu- 
ally refers to displeasure. It consists of subjective feelings that vary in intensity, from mild irritation or annoyance to intense fury and rage. It also implies destructive behaviours toward other persons or objects (Spielberger, Jacobs, Russell, \& Crane, 1983; Spielberger, Reheiser, \& Sydeman, 1995; Ramírez \& Andreu, 2006)

Measures of anger can serve as predictors of aggressive behaviour (Harmon-Jones, 2003; Swaim, Henry, \& Kelly, 2006) in adults, adolescents, and children (Campano \& Munakata, 2004; Underwood, 2005). Reactions of anger include confronting injustice or frustration (Spielberger, et al., 1983; Ramírez, Fujihara, \& van Goozen, 2001). It is suggested that anger increases if the source is perceived as being intentional, preventable, or unjustified, and when values are compromised, promises and expectations are broken, rules violated, or personal freedom and rights are abridged (Levine, 1995). Anger is regularly considered a negative emotion due to its possible harmful consequences, e.g., aggression (Lazarus, 1991). But it can also be associated with prosocial actions regulated through moral codes that address situations considered socially unfair (Cox, Lopez, \& Schneider, 2003; Haidt, 2003; Harmon-Jones, Sigelman, Bohlig, \& Harmon-Jones, 2003; Moll, de OliveiraSouza, Moll, Ignacio, Bramati, Caparelli-Daquer, \& Eslinger, 2005), so that it is viable to consider anger not only a basic but also a social and moral emotion that requires a theory of mind to infer others mental, emotional, and physical states (Haidt, 2003).

The definition of the elements and particular elicitors of one emotion becomes difficult when considering that individuals could experience negative emotions, such as anger, fear, or sadness if they fail to maintain something valuable or are confronted with unpleasant stimuli (Mikels, et al., 2005), such as a visual scene of attach (Bradley, Codispoti, Cuthbert, \& Lang, 2001; Bradley, Codispoti, Sabatinelli, \& Lang, 2001). Also, the experience of anger, fear, and sadness elicits similar electromyographic activity (Hu \& Wan, 2003). The formal differences in the experiences of anger and fear are not clear, although one seems to be that fear leads to risk aversion, while anger leads to risk seeking (Lerner \& Keltner, 2001). When considering anger and sadness, both negative emotions could be elicited with the occurrence of negative events, e.g., blaming others and loss (Abramson, Metalsky, \& Alloy, 1989; Smith \& Lazarus, 1993). Hence, anger and sadness may be differentiated from each other only in how the events are being appraised: people can feel angry when they have a negative experience that is attributed to another person who had control over what happened (Weiner, Graham, \& Chandler, 1982; Averill, 1983; Ellsworth \& Smith, 1988; Levine, 1996), while sadness may be a response to negative events that are characterized by rejection and interpersonal loss (Robins \& Block, 1988; Levine, 1996; Buss \& Kiel, 2004). 
Anger and sadness differ in their motivational intensity. For example, infants who express anger during an extinction process maintain interest during subsequent relearning, whereas those who express sadness show decreased interest in relearning (Lewis, Sullivan, Ramsey, \& Alessandri, 1992). In terms of social communication, expressions of sadness may be more beneficial than the other emotions for eliciting helping behaviour in others (Buss $\&$ Kiel, 2004). The more obvious elicitors of the three emotions are loss eliciting sadness, cheating or harm eliciting anger, and danger or threat eliciting fear.

Because recognition of an expression includes a cognitive evaluation of the stimulus, identifying an emotion is not sufficient for understanding why the individual rates an expression as having specific intensity in valence or arousal (Lazarus, 1991; Damasio, 1996; Moll, de Oliveira-Souza, Eslinger, Bramati, Mourao-Miranda, Andreiuolo, \& Pessoa, 2002; Haidt, 2003; Eisenberg, et al., 2005). The perception and identification of facial expressions are influenced by subjective attribution (Bimler \& Paramei, 2006). This suggests that the information about the context of a stimulus is evaluated for its relevance to multiple concerns for the organism, and human affect is involved in social control through behavioural signals regulating expressions and gestures (Fridja, 1986, 1996).

Such complex social and subjective factors require a cognitive system for processing emotions. Damasio (1996) argued that complex emotions involve differentiated somatic appraisals and cognitive activity. The somatic signals conjoined to social development and experiences make possible decisions in each specific emotional circumstance, and may explain the frequent misattributions people make. Conversely, other authors suggest that emotion and cognition are independent systems (Murphy \& Zajonc, 1993). They argue that some emotions are associated with sensations such as fear but others are not-for example, pride or remorse. Emotions are elicited not only by physical stimuli. For instance, fear can be experienced when one thinks of something frightening or harmful, such as a possible accident. The cognitive factor of emotion does not always include appetitive motivation; for instance, the sensation of hunger is related to looking for food but not with any particular beliefs (Bennett \& Hacker, 2005).

The two main objectives of this study were to define the contextual and dimensional elements included in visual stimuli evoking anger and to identify the presence of other negative emotions related to an individual's experience of anger, particularly sadness and fear. One common method of studying the identification of expressions and appraisals related to single emotions is responding to validated scales while viewing pictures of emotionally charged scenes (Lang, Greenwald, Bradley, \& Hamm, 1993; Bradley, Codispoti, Cuthbert, \& Lang, 2001; Bradley, Codispoti, Sabatinelli, \& Lang, 2001; 
Mikels, et al., 2005). According to previous literature, it was hypothesised that the most intense rating of Anger on the Self-Assessment Manikin would be evoked by International Affective Picture System pictures showing an Intention to Damage or to Harm, followed by those representing Frustrated Goals and Angry Expressions. The experience of Anger would be correlated with the experience of other emotions such as Fear and Sadness. Being considered a negative emotion, Anger was expected to be positively correlated with low Valence, high Arousal, and high Dominance. Finally, sex differences were expected since women express their emotions more and recognize facial expressions better than men (Levenson, Carstensen, Friesen, \& Ekman, 1991; Stroebe, 2001; LaFrance, Hecht, \& Paluck, 2003; Sternglanz \& DePaulo, 2004; Rhudy \& Williams, 2005).

\section{Participants}

METHOD

An invitation to adult volunteers ( 25 to 30 yr. of age) to participate in research on emotions was presented to students of psychology at a university in Madrid. Forty-five subjects (25 women and 20 men: $M$ age $=27.2$ yr., $S D$ $=9.5$ ) were selected. General health and absence of psychiatric and neurological antecedents and symptoms were verified through a semistructured interview; no volunteer was taking any medication during the experiment. Informed consent was obtained after the nature of the experiment had been explained to the volunteers. They were not paid for their participation. The design of the experiment was made in accordance with the ethical principles recommended by the American Psychological Association (O'Donohue \& Ferguson, 2003).

\section{Visual Materials}

According to the kind of stimulus eliciting anger reported in previous studies, a set of 120 pictures was selected from the International Affective Picture System (IAPS), which constitutes a large collection of photographs emotionally calibrated on three bipolar motivational axes. Valence (pleasantunpleasant), Arousal (motivational activation), and Dominance (control over the depicted situation; Lang, Bradley, \& Cuthbert, 2005). Each picture belonged to one of four categories: (a) 48 pictures showing Intention to Harm, for example, assaults, women being knocked down, and animals being mutilated; (b) 48 pictures showing Frustration of Goals, for example, people in jail and children in war; (c) 12 pictures showing Anger Expressions without explicit social contexts; and (d) 12 Neutral pictures, for example, people walking on the street and people drinking coffee.

\section{Appraisal Materials}

The paper-and-pencil version of the Self-Assessment Manikin (Lang, et al., 2005) was used to rate each picture. This scale is suggested for evalua- 
tion of the pictures from the IAPS. The Self-Assessment Manikin is an affective rating scale for self-assessment of appraisals along three bipolar dimensions: Valence, Arousal, and Dominance. Each dimension is represented by a graphic figure depicting nine marks, where the first mark (score=1) represents the lowest rating on each dimension (viz., low pleasure or unpleasant, low arousal or calm, and low dominance or controlled) and the last mark (score $=9$ ) represents the highest rating (viz., high pleasure or pleasant, high arousal or excited, high dominance). In order to measure the three emotions of interest, three emotion scores were added to the Self-Assessment Manikin scales, namely Anger, Sadness, and Fear. Similar to the Self-Assessment Manikin, subjects were asked to write the intensity of their experience of these three emotions using a 9-point scale, where a score of 1 represents a usual emotional experience and a score of 9 the most intense experience of the emotion.

Procedure

The experiment was designed according to the International Affective Picture System Instruction Manual and Affective Ratings (Lang, et al,, 2005). The 120 pictures were randomized and presented in a 32-bit colour resolution of 1,000 by 750 pixels. The sessions were conducted in a room of 9 by $10 \mathrm{~m}$ under uniform lighting conditions. Participants were seated in rows at 90 degrees to a $3-\mathrm{m}$ by $2-\mathrm{m}$ screen where the slides were projected. The projection was controlled by a computer through a serial port. Each experimental session included a group of 15 subjects.

Before the slide presentation, participants were instructed how to rate the dimensions and the emotions in their paper sheet. Each participant rated each picture according to the instructions given. Five test slides, were shown to ensure the proper understanding of the task. The trials began with a 5 -sec. presentation of a preparatory slide that indicated the number of the next picture to be presented. Then the picture to be rated was shown for $6 \mathrm{sec}$. Immediately after the picture left the screen, a slide presented for $20 \mathrm{sec}$. instructed participants to answer the scales. Each complete session lasted 60 $\min$.

\section{Statistical Analyses}

Ratings of Valence, Arousal, and Dominance obtained in this study were compared with those reported in the original American database (Lang, et al., 2005) and in the Spanish normalization (Moltó, Montañés, Poy, Segarra, Pastor, \& Tormo, 1999; Vila, Sánchez, Ramírez, Fernández, Cobos, Rodríguez, et al., 2001) of the pictures included in the IAPS. A Student $t$ test was used to compare the mean of ratings of the pictures in each category (Intention to Harm, Frustration of Goals, Anger Expressions, and Neutral).

The data have a doubly multivariate structure, with measurements on 
six (correlated) emotional experiences across 120 images for each participant. Two types of analysis were applied: one based on a parametric model from the family of mixed effects models and the other based on a nonparametric three-way model from the family of hierarchical classes models. Both methods explicitly account for the doubly multivariate structure in the data, but do so in radically different ways and, as a result, highlight different aspects of the data.

Linear mixed model approach.-For the sake of simplicity in both the specification of the fitted model and the interpretation of the results, prior to the analysis, the 120 data points for each emotional experience (Valence, Arousal, Dominance, Anger, Sadness, Fear) of each participant were standardized to a mean of 0 and a variance of 1 . This transformation removes differences among subjects due to response tendencies such as selecting extreme versus moderate positions, i.e., extreme or moderate response bias, and shifting responses to either end of the scale, i.e., acquiescence response bias. Furthermore, it brings the six emotional dimensions to a common scale with equal mean and variance. Subsequently, the following mixed effects model was fitted to the data (for an introduction, see Vonesh \& Chinchilli, 1997). Equation [1] represents the analysis used, where IH refers to the Intention to Harm variable, FG to Frustration of Goals, AEX to Anger Expressions and NEU refers to Neutral pictures:

$$
Y_{i j k}=\mu_{k}^{\mathrm{II}} x_{j}^{\mathrm{IH}}+\mu_{k}^{\mathrm{FG}} x_{j}^{\mathrm{FG}}+\mu_{k}^{\mathrm{AEX}} x_{j}^{\mathrm{AEX}}+\mu_{k} \mathrm{NEU}_{x_{j}}^{\mathrm{NEU}}+u_{j}+\varepsilon_{i j k},
$$

with $u_{j} \underset{\sim \text { iid }}{\sim} \mathrm{N}\left(0, \sigma_{u}^{2}\right),\left(\varepsilon_{i j 1}, \ldots, \varepsilon_{i j 6}\right)^{\prime} \stackrel{\text { iid }}{\sim} \mathrm{N}\left(0, \Sigma_{\varepsilon}\right)$ and $\Sigma_{\varepsilon}$ being a variance-covariance matrix, restricted to be positive definite. In Equation [1] above, the dependent variable $Y_{i j k}$ denotes the standardized value of subject $i(1 \leq I \leq 45)$ on emotional dimension $k(1 \leq k \leq 6)$ while observing image $j(1 \leq j \leq 120)$; the $X_{j}^{b}$ ( $\mathrm{h}=$ Intention to Harm, Frustration of Goals, Anger Expressions, Neutral pictures) are binary dummy variables indicating the a priori category to which image $j$ belongs, such that the $\mu_{k}^{b}$ represents the mean intensity on emotional dimension $k$ across pictures of category $b$. The random effects uj account for differences among images not explained by the category they belong to and the $\varepsilon_{i j k}$ account for the residual variance. Note that $\Sigma_{\varepsilon}$ accounts for the covariances, assumed to be identical across persons and images, among the six emotional dimensions within person $i$ and image $j$.

Hierarchical Classes Approach

Hierarchical classes (HICLAS) models are a family of models for the analysis of two-way (De Boeck \& Rosenberg, 1988; Van Mechelen, de Boeck, \& Rosenberg, 1995) or three-way data (Leenen, van Mechelen, de Boeck, \& Rosenberg, 1999; Ceulemans, van Mechelen, \& Leenen, 2003). The current data are three-way with 45 [(subjects) $\times 120$ (images) $\times 6$ (emotional dimensions)] data points. 
All models in the HICLAS family share three common features: (a) a classification of the elements in each way, i.e., a classification of subjects, a classification of images, and a classification of emotional experiences; (b) the classes of elements are hierarchically organized, where the hierarchies can be interpreted as if-then rules; (c) the hierarchies for each way are linked together, as such yielding a predicted or reconstructed data array, e.g., indicating which emotions or emotional dimensions a subject experiences while observing a given image. Tucker3-HICLAS analysis (Ceulemans, et al., 2003) was applied to the three-way data in this study. This type of analysis, like most HICLAS analysis, requires a binary data array as input. So as not to lose too much information, it was decided to recode the ratings for each emotional dimension in three categories by means of two binary variables, indicating a moderate emotional experience (at least a score of 4 on the 9point scale) and strong emotional experience (at least a score of 7). The Tucker3-HICLAS model goes with a comprehensive graphical representation, from which the three HICLAS features can be directly read. We will discuss the interpretation of the graphical representation when we present the model resulting from the analysis in the next section.

\section{Results}

According to the Student $t$ test analysis, ratings obtained in the Valence, Arousal, and Dominance emotional dimensions were not different from those reported in the Spanish normalization of the pictures included in the IAPS. Nevertheless, Valence reported in the pictures included in the category Frustration of Goals was lower than the original American reports $\left(t_{94}=\right.$ $-1.46, p<.05)$. Also in this category, Arousal reported in this study was higher than that reported in the original American data $\left(t_{94}=2.29, p<.01\right)$.

\section{Linear Mixed Model Approach}

Each estimate corresponds directly to the mean intensity of an emotional experience across pictures from a given a priori category. The size of the differences can be readily interpreted in terms of units of standard deviations, as a result of the standardization prior to the analysis (Table 1).

The estimates for the variance components are presented in Table 2. First, the significant value for the variance $\sigma_{u}^{2}$ means that pictures of the same category also generally evoke different intensities on the emotional dimensions. Second, the variance-covariance matrix $\Sigma_{\varepsilon}$ shows that there is still considerable covariation left among the emotional dimensions, after covariation due to differences among people and differences among pictures has been accounted for. That is, $\Sigma_{\varepsilon}$ shows how emotional dimensions covary within a person observing some image. So as to get a better understanding of the relation between, on the one hand, the dimensions of Valence, Arous- 
TABLE 1

Estimates of Fixed Effects in Mrxed Model Equation [1] Concerning Three Emotions (Anger, Fear, Sadness) and Three Emotional Dimensions (Valence, Arousal, Dominance) in Four Categories of Pictures Evaluated

\begin{tabular}{|c|c|c|c|c|}
\hline \multirow{2}{*}{$\begin{array}{l}\text { Emotion and } \\
\text { Dimension }\end{array}$} & \multicolumn{4}{|c|}{ Category of Pictures } \\
\hline & $\begin{array}{l}\text { Intention to } \\
\text { Harm }\end{array}$ & $\begin{array}{c}\text { Frustration of } \\
\text { Goals }\end{array}$ & $\begin{array}{c}\text { Anger } \\
\text { Expressions }\end{array}$ & Neutral \\
\hline Anger & $0.44^{\mathrm{d}}$ & $-0.05^{\mathrm{c}}$ & $-0.61^{\mathrm{b}}$ & $-0.94^{\mathrm{a}}$ \\
\hline Fear & $0.41^{\mathrm{d}}$ & $-0.11^{c}$ & $-0.42^{b}$ & $-0.77^{\mathrm{a}}$ \\
\hline Sadness & $-0.08^{b}$ & $0.57^{c}$ & $-0.86^{\mathrm{a}}$ & $-1.11^{\mathrm{a}}$ \\
\hline Valence & $0.26^{\mathrm{c}}$ & $0.33^{c}$ & $-0.62^{b}$ & $-1.72^{a}$ \\
\hline Arousal & $-0.28^{\mathrm{a}}$ & $-0.17^{a}$ & $0.79^{b}$ & $1.02^{b}$ \\
\hline Dominance & $-0.16^{a}$ & $-0.18^{a}$ & $0.55^{b}$ & $0.80^{\mathrm{b}}$ \\
\hline
\end{tabular}

Note-Effects in the same row with different superscripts are significantly different $(p<.05$, Tukey-adjusted).

al, and Dominance, and, on the other hand, the emotions of Anger, Fear, and Sadness, we fitted a path model to the variance-covariance matrix $\Sigma_{\varepsilon}$ (see, e.g., Kline, 2005).

TABLE 2

Variance Components Estimates in Mixnd Model Equation [1] Concerning Three Emotions (Anger, Frar, Sadness) and Three Emotional Dimensions (Valence, Arousal, Dominance)

\begin{tabular}{lccccccc}
\hline \hline$\Sigma_{\varepsilon}$ : Emotion and Dimension & 1 & 2 & 3 & 4 & 5 & 6 \\
\hline 1. Anger & 0.75 & 0.20 & 0.26 & 0.23 & -0.34 & -0.21 \\
2. Fear & 0.25 & 0.82 & 0.07 & 0.16 & -0.32 & -0.28 \\
3. Sadness & 0.38 & 0.10 & 0.63 & 0.28 & -0.29 & -0.25 \\
4. Valence & 0.36 & 0.24 & 0.47 & 0.55 & -0.27 & -0.25 \\
5. Arousal & -0.44 & -0.40 & -0.41 & -0.40 & 0.79 & 0.40 \\
6. Dominance & -0.26 & -0.34 & -0.35 & -0.37 & 0.48 & 0.86 \\
\hline
\end{tabular}

Note $-\sigma_{u}^{2}=0.015(p<.01)$. The diagonal, upper and lower triangles show variances, covariances, and correlations, respectively. All parametêrs are significantly different from $0(p<01)$.

Paths from any of the former dimensions to any of the latter emotions were specified because Valence, Arousal, and Dominance might be considered to be in some sense a priori to Anger, Fear, and Sadness (see Fig, 1). Note that the fitted model is just identified, and as such perfectly predicts $\Sigma_{\varepsilon}$. Interestingly, Valence is found to have a positive effect on each of the three emotions, while the effect of Arousal and Dominance on Anger, Fear, and Sadness is negative.

Hierarcbical Classes Approach

In a typical Tucker3-HICLAS analysis, models are fitted to the same data set. These models differ from each other with respect to complexity and goodness of fit to the data. A model is then selected for further discussion by looking for an optimal trade-off between parsimony or complexity 


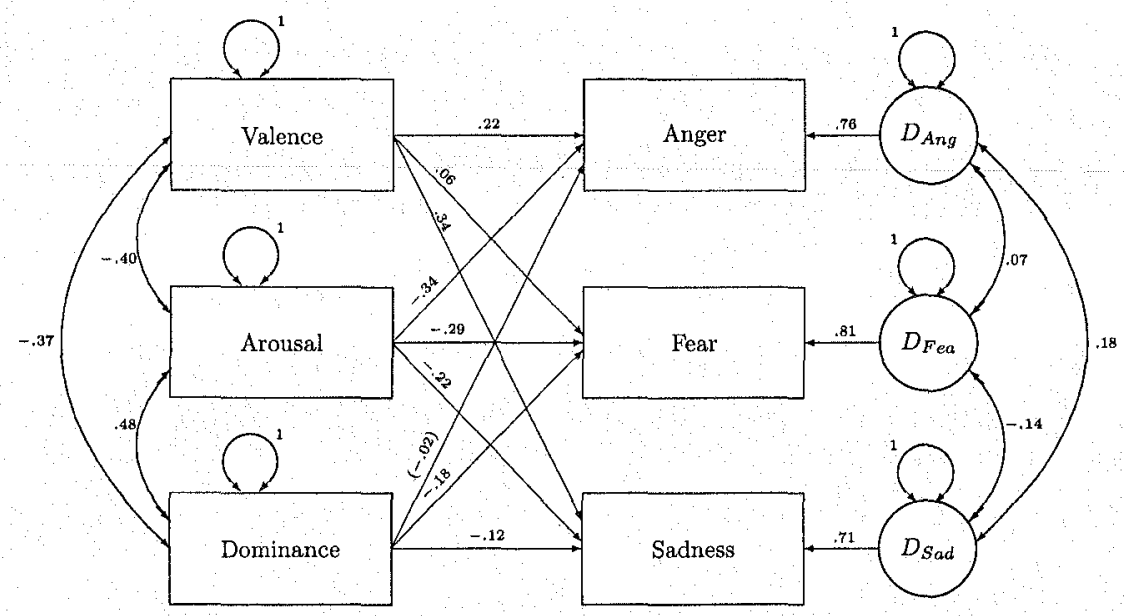

FIG, 1. A just-identified path model for the covariance matrix $\Sigma_{\text {. Standardized estimates }}$ are presented; estimates for the disturbances are proportions of unexplained variance. Estimates that are not significantly different from 0 are placed between parentheses.

and goodness of fit. This practice is similar to practices in principal components analysis and most clustering techniques, where one typically looks for a model with a small number of components or clusters that at the same time is as least discrepant as possible with the data. The complexity of a Tucker3-HICLAS model is given by its rank-a triple of integers which fix the maximum number of classes for each of the three classifications-while goodness of fit is usually expressed by the proportion of binary data points that are mispredicted by the model. Several heuristics, which often bare resemblance with similar heuristics in factor analysis such as Cattell's (1996) scree test, have been developed for model selection in hierarchical classes analysis.

For the current data, models of rank $(1,1,1)$ to $(5,5,5)$ were fitted. Based on a combination of substantive (i.e., easy interpretation) and formal criteria (viz, rank vs fit trade-off), the model of rank $(2,3,3)$ was selected for further discussion. The graphical representation of this model is shown in Fig. 2.

The upper half of Fig. 2 represents the hierarchical classification of the pictures, with each box corresponding to a class of pictures. Within each box, the number of pictures from each category is indicated (Intention to Harm, IH; Frustration of Goals, FG; Anger Expressions, AG; and Neutral, NEU) belonging to the class. Pictures that belong to the same class evoke the same set of emotional experiences within each person. For example, it was observed that the 12 Neutral (NEU) pictures and the 12 Anger Expres- 


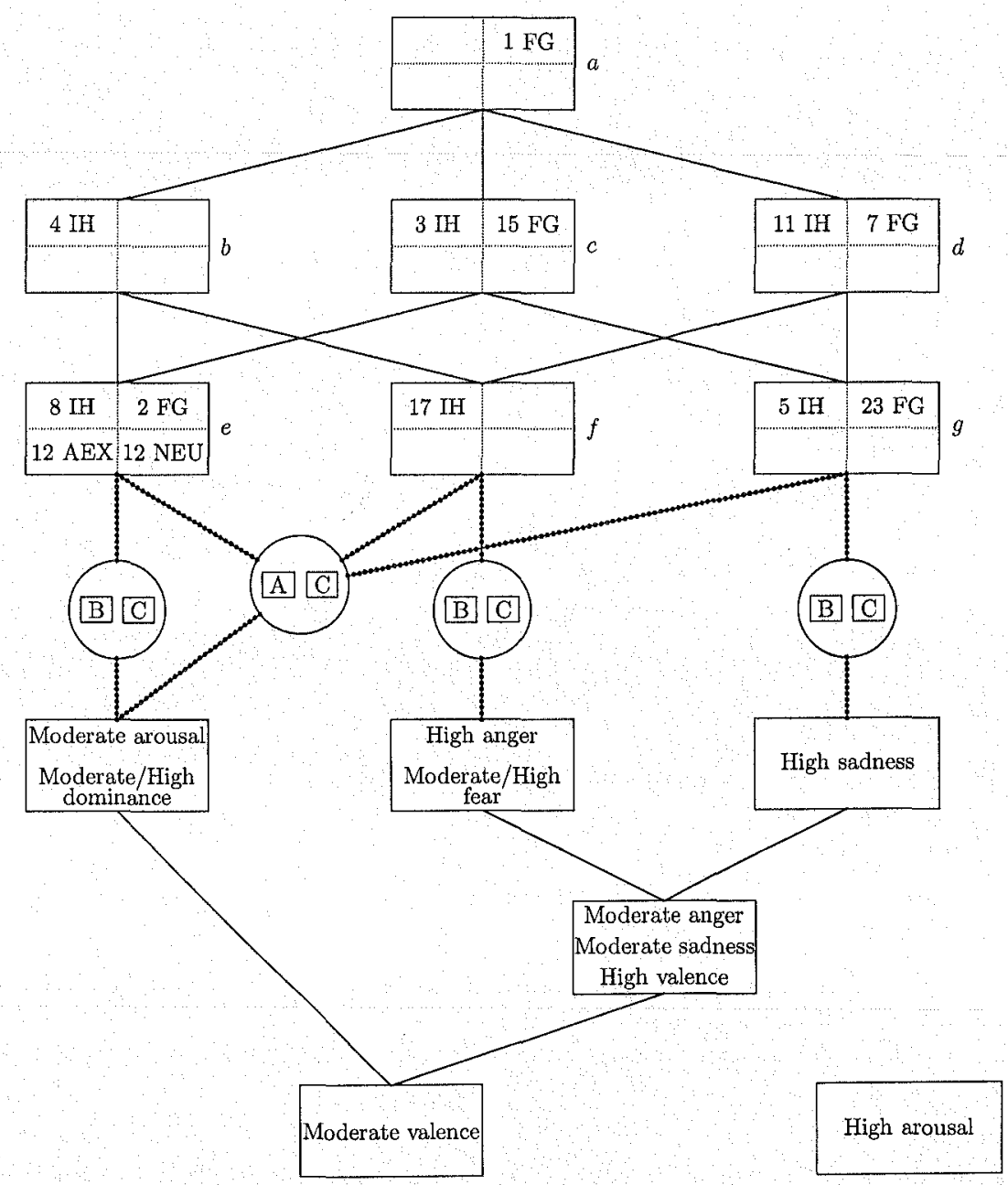

FIG. 2. Rank $(2,3,3)$ Tucker3-HICLAS model for the three-way dichotomized data array. The capitals $A, B$, and $C$ in the circles at the center of the figure denote different person classes. The classes a to $\mathrm{g}$ in the image (top half) show the number of pictures showing Intention to Harm (IH), Frustration of Goals (FG), Anger Expressions (AEX), and Neutral images (NEU).

sions $(\mathrm{AEX})$ pictures all appeared in a single class together with 10 pictures of the two other categories, and hence, evoked the same emotional experiences. For most other classes (except the class labeled $d$ ), a clear majority of either the Intention to Harm (IH) pictures or Frustration of Goals (FG) pictures were noted, which can be considered a validation of the a priori cate- 
gorization of these pictures. Hierarchical relations among the classes are represented by lines connecting the boxes. For example, class $d$ is hierarchically above class $g$, which means that any emotional experience evoked by pictures in class $g$ will be evoked by pictures in class $d$ as well. This implies too, for example, that the picture in class $a$ evokes any feeling that any other picture evokes.

The lower half of the figure contains the hierarchical classification of the emotional dimensions. Emotions that appear together in a class are simultaneously experienced within persons. For example, high Anger was experienced with moderate and high Fear. Again, the hierarchical relations among the emotional experiences are represented by straight lines connecting the boxes, but for reasons that will become clear in a moment, the hierarchy is represented upside down. For example, the class with high Anger and moderate and high Fear is hierarchically below the class of moderate Anger, moderate Sadness, and high Valence, which means that if a person experiences the former emotions, then he will experience the latter as well. Note that high Arousal is not connected to any of the other boxes; this is because high Arousal was not experienced by any person in response to any of the pictures (i.e., only moderate Arousal was reported).

The model includes a classification of the 45 participants into three classes, represented by the capital letters in the center of the figure. Persons who belong to the same class reported the same emotional dimensions in response to any particular picture. The hierarchical relations among the person classes are not represented in Fig. 2, but are shown separately in Fig. 3. If a person is placed hierarchically above another person, then the former reported on the emotional dimensions at least at the same level as the other person while viewing any particular picture (i.e., if the other person reported some emotion, then the person reported that emotion at the same or higher level). As a result, according to sex, the persons in class $C$ can be considered most sensitive, because they reported all emotions that the persons of the two other classes reported at the same or higher level.

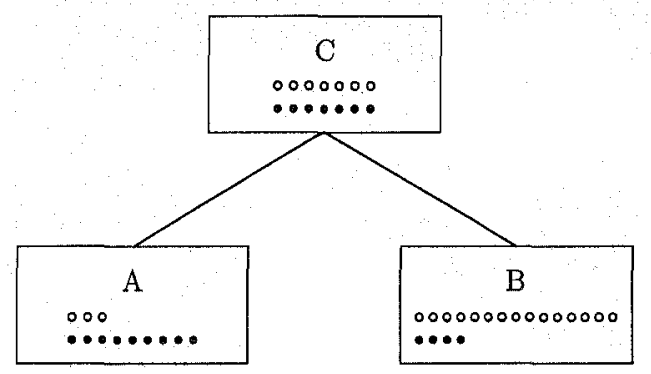

Fig. 3. Hierarchical classification of the persons. The number of dots in a class indicates how many women $(0)$ and men $(\bullet)$ belong to that class. 
The Tucker3-HICLAS model defines an association relation linking the three hierarchical classifications (pictures, emotional experiences, and persons) together. This linking structure is represented by the connections in the center of Fig. 2, which allow the reader to derive whether a given person (class) experiences a given emotional dimension (class) while observing a given picture (class). In particular, if a downward path can be found that connects a given picture (class) to a given emotional dimension (class) passing by a circle containing the person (class), then this person reported this emotional dimension when observing this picture. For example, persons from class $A$, with a majority of men, reported high Dominance on all pictures: the class containing the dimension of Dominance can be reached from all picture classes while passing by person class A. In contrast, persons from class $B$, with a majority of women, only reported Dominance when observing pictures from classes $a, b, c$, and $e$, which, amongst others, contain Anger Expressions and Neutral pictures. On the other hand, persons from class A never reported experiences of Anger or Fear, so there is no connection between this person class and these emotions, whereas persons from class B reported high Anger and Fear to a considerable number of pictures, amongst which are $75 \%$ of the Intention to Harm pictures. These examples show how the hierarchical classes approach offers a valuable tool for studying the relations among pictures, emotional experiences, and persons.

Discussion

Comparisons of the ratings of Valence, Arousal, and Dominance obtained in this study with those reported in the original American database and the Spanish normalization of the IAPS confirm that scores in Spanish and American populations are similar, but Spanish samples tend to assign higher Arousal ratings (Moltó, et al., 1999; Vila, et al., 2001).

Participants reported that most of the pictures evoking Anger elicited Fear and/or Sadness too. This leads to the inference that Anger is not only a basic emotion, but also a complex one that shares its experience with other emotions depending on the context shown in the stimulus and the subjective attribution (Bimler \& Paramei, 2006). Pictures evoking moderate Anger and Sadness were reported with similar experiences of Valence, as did pictures evoking high Anger and high Fear, probably because the three emotions are elicited when confronted with unpleasant stimuli (Mikels, et al., 2005). Nevertheless, high Anger and high Fear overlapped in the same class, so that unpleasant experiences associated with Anger are closely related to stimuli evoking Fear, but not with those evoking Sadness, maybe because both Anger and Fear are related to a strong motivational system of aversion related to risk elements (Lerner \& Keltner, 2001).

Any picture was associated with high Arousal. The experience of high 
Arousal or excitation permits the understanding of emotions as part of motivational and developmental systems that help organisms avoid dangerous or aversive stimuli (Plutchik, 1980). It would make sense that the experience of Anger and Fear would be associated with high Arousal. Nevertheless, results indicate an association with the experience of moderate Arousal. This may be due to the fact that those motivational and developmental systems function in real situations that affect the individual's life, which are difficult to reproduce in experimental conditions and perhaps not elicited at all by watching pictures. Some physiological variables implicated in emotional responses to real situations have been observed in laboratory conditions, e.g., electromyographic activity (Bradley, Codispoti, Cuthbert, \& Lang, 2001; Bradley, Codispoti, Sabatinelli, \& Lang, 2001; Hu \& Wan, 2003). The emotional experiences reported in this and similar studies refer to a subjective process dependent on emotion and cognition (Damasio, 1996), which could lack appetitive motivations (Bennett \& Hacker, 2005). The procedure for evaluation of pictures was executed in groups of several persons in a room with controlled environmental conditions, so it is also necessary to consider in future studies the presence of other people in the place of evaluation who may influence each other's emotional expressions and responses.

A similar interpretation could explain the experience of Dominance. Contrary to the expectation of being associated with experiences of Anger, appraisals of Dominance were associated with the experiences of Anger, Fear, and Sadness. This may be attributed to the artificial social situations represented by looking at the pictures.

As expected, those pictures that were more robust elicitors of Anger showed Intention to Harm, followed by pictures showing Frustration of Goals. Sadness was mostly elicited for pictures showing Frustration of Goals, followed by pictures showing Intention to Harm. Similar to Anger, Fear was strongly elicited by pictures showing Intention to Harm.

These previous results suggest that anger may be strongly elicited by the observation of damage or harm caused intentionally by a person who has control over the situation (Averill, 1983; Levine, 1995; Ramírez, et al., 2001). Thus, anger can be considered a social and moral emotion that implies the inference of the emotional and physical state of others provoked by a norm violation (Haidt, 2003). Alternatively, the fact that Fear and Anger were both strongly elicited by pictures in the category Intention to Harm may reflect that both emotions include processes of theory of mind. Fear evoked by Intention to harm pictures suggests that the observer infers the possibility of being in a similar situation, even if this situation is hypothetical. In this case, the emotional experience does not always require a formal object (Bennett \& Hacker, 2005).

On the other hand, the fact that Sadness was elicited by pictures in the 
category Frustration of Goals but not in Intention to harm may imply that the experience of Sadness is more related to the empathy elicited by interpersonal loss or pain in others, even if the cause of the event was not explicit. According to previous reports, sadness but not anger is related to rejection and interpersonal loss, and is an effective communicative social system (Robins \& Block, 1988; Levine, 1996; Buss \& Kiel, 2004).

Observing emotional facial expressions allows the identification of another person's physical or psychological states. The observation of pictures showing Anger Expressions did not elicit Anger. Although previous studies (Fernández-Dols \& Ruíz-Belda, 1997) reported that the observation of facial expressions might evoke similar motor expressions-observing people smiling evoked similar smiling as a rection-it did not elicit the similar emotional experience. This may reflect that, aside from facial expressions, emotional experience requires a complete processing of the circumstances, which includes the interpersonal and social context and the inference of others' psychological and physical states. Hence, emotional experience implies cognitive and affective systems acting concurrently to process a stimulus (Damasio, 1996).

Anger, Fear, and Sadness were differently appraised for each category of pictures, except that Sadness was elicited equally for pictures representing Anger Expressions and for Neutral pictures (see Table 1). This suggests that, even if the experience of the three emotions overlap in some cases, it is also possible to define specific features of the stimulus evoking them, in accord with the categorical model for studying emotions (Izard, 1992; Ekman, 1999). Equal intensity in experiencing Valence, Arousal, and Dominace was reported for pictures representing Intention to Harm and Frustration of Goals. However, experiences of Arousal and Dominance were similar in pictures representing Anger Expressions and Neutral pictures. Therefore, relationships among emotions and emotional dimensions are not linear. Results also permit the inference that even though Anger, Sadness, and Fear have been well classified as basic emotions, they are elicited by social contexts that may require complex processing prior to or simultaneous with that experience. It also suggests that similar appraisals are shared by different emotions so that an approach based only on a discrete model could be used for classifying the kind of elicitors of one emotion, but not for defining their particular subjective components.

The correlation of Anger, Sadness, and Fear with low Valence, high Arousal, and high Dominance in some categories of pictures (see Table 2) illustrate the typical attributions of negative emotions (Cuthbert, et al., 1996; Eisenberg, et al., 2005; Mikels, et al., 2005). These results suggest that the dimensional approach for studying emotions can identify subjective components of one emotion but not classify it completely. 
As expected, sex differences in responses were found. More men than women reported higher Dominance in all pictures, but more women than men did not report Dominance in several pictures representing Intention to Harm and Frustration of Goals. This explains that women report less control when observing the features depicted in those categories of pictures. Experiences of high Valence, Anger, or Fear and Sadness were reported more for women than for men. These results replicate the finding that women are more likely to report emotions as intense (Levenson, et al., 1991; Stroebe, 2001; LaFrance, et al., 2003; Sternglanz \& DePaulo, 2004; Rhudy \& Williams, 2005).

Taking into consideration the statistical model used in this work, persons from class A ( 3 women and 9 men) never reported experiences of Anger or Fear (there is no connection between this person class and these emotions), whereas persons from class B (15 women and 4 men) reported high Anger and Fear to a considerable set of pictures, amongst which were $75 \%$ of the Intention to Harm category (see Figs. 1 and 3). These examples represent how the hierarchical classes approach offers a valuable tool for studying the relations among pictures, emotional experiences, and persons.

The present results suggest that the study of emotions requires a model which combines the discrete and dimensional approaches in order to distinguish the elicitors and the subjective experiences related to a particular emotion. Even if anger is a basic and discrete emotion, its experience is also shared with other emotions. Consequently, its study, instead of being directed toward one specific emotion, must focus on a global emotional experience more closely associated with real situations. When studying emotions, therefore, it would be useful to consider simultaneously the consistency between three elements: (a) evaluation of the subjective experience by using appraisals and reports based on the explanation of what a discrete emotion is; (b) correlation of physiological reactions, such as heart rate or galvanic skin response, with the subjective experience; and (c) recording and classification of the facial expressions shown in response to emotional pictures.

\section{REFERENCES}

Abramson, L. Y., Metalsky, G. I., \& Alloy, L. B. (1989) Hopelessness depression: a theorybased subtype of depression. Psychological Review, 96, 358-372.

Averill, J. R. (1983) Studies on anger and aggression: implications for theories of emotion. American Psychologist, 38, 1145-1160.

Avero, P., \& Calvo, M. G. (2006) Affective priming with pictures of emotional scenes: the role of perceptual similarity and category relatedness. Spanish Journal of Psycbology, 9, 10-18.

BeNNETT, M. R., \& HaCker, P. M. (2005) Emotion and cortical-subcortical function: conceptual developments. Progress in Neurobiology, 75, $29-52$.

Bimler, D. L., \& Paramei, G. V. (2006) Facial-expression affective attributes and their configural correlates: components and categories. Spanish Joumal of Psychology, 9, 19-31.

Bradley, M. M., Codispoti, M., Curterbert, B. N., \& Lang, P. J. (2001) Emotion and motivation: I. Defensive and appetitive reactions in picture processing. Emotion, 1, 276-298. 
Bradley, M. M. Codispoti, M., Sabatinelli, D., \& Lang, P. J. (2001) Emotion and motivation: II. Sex differences in picture processing. Emotion, 1,300-319.

Buss, K. A., \& Kiel, E. J. (2004) Comparison of sadness, anger, and fear facial expressions when toddlers look at their mothers. Child Development, 75, 1761-1773.

Campano, J. P., \& Munakata, T. (2004) Anger and aggression among Filipino students. Adolescence, $39,757-764$

Cattell, R. B. (1996) The meaning and strategic use of factor analysis. In R. B. Cattell (Ed.), Handbook of multivariate experimental psychology. Chicago, IL: Rand McNally. Pp. 174243.

Ceulemans, E., van Mrchelem, I., \& Leenen, I. (2003) Tucker3 hierarchical classes analysis. Psychometrika, 68, 413-433.

Cox, R. L., Lopez, N. L., \& Schnerder, H. G. (2003) Anger expression: parental and cognitive factors. Psychological Reports, 93, 59-65.

Cuthbert, B. N., Bradley, M. M., \& Lang, P. J. (1996) Probing picture perception: activation and emotion. Psychopbysiology, 33, 103-111.

Damasio, A. (1996) El error de Descartes: la razón de las emociones. Santiago de Chile: Andrés Bello,

Darwin, C. (1988) La expresión de las emociones en los animales y en el bombre (T. R. Fennández, Transl.) México: Alianza Editorial Mexicana. [Original published in 1872]

De Bozck, P., \& Rosenberg, S. (1988) Hierarchical classes: model and data analysis. Psychometrika, 53, 361-381.

DíAz, J. L., \& Flores, E. (2001) Le estructura de la emoción humana: un modelo cromático del sistema afectivo. Salud Mental, 24, 20-35.

Etsenjberg, N., SADovsky, A, \& SpinRAd, T. L. (2005) Associations of emotion-related regulation with language skills, emotion knowledge, and academic outcomes. New Directions for Child and Adolescent Development, 109, 109-118.

EkMan, P. (1999) Basic emotions. In T. Dalgleish \& T. Power (Eds.), Handbook of cognition and emotion. Sussex, UK: Wiley. Pp. 45-60.

Ellsworth, P. C., \& SMITH, C. A. (1988) From appraisal to emotion: differences among unpleasant feelings. Motivation and Emotion, 12, 271-302.

Fernández-Dols, J. M., \& Rứz-Belda, M. A. (1997) Spontaneous facial behavior during intense emotional episodes: artistic truth and optical truth. In J. A. Russell \& J. M. Fernández-Dols (Eds.), The psycbology of facial expressions. Cambridge, UK: Cambridge Univer. Press. Pp. 255-294.

FridjA, N. H. (1986) The emotions. New York: Cambridge Univer. Press.

FRIDJA, N. H. (1996) Passions: emotion and socially consequential behavior. In R D. Kavanaugh, B. Zinmerberg, \& S. Fein (Eds.), Emotion: interdisciplinary perspectives. Mahwah, NJ: Erlbaum. Pp. $1-28$

HaidT, J. (2003) The moral emotions. In R. J. Davidson, K. Scherer, \& H. Goldsmith (Eds.), Handbook of affective sciences. Oxford, UK: Oxford Univer. Press. Pp. 852-870

Harmon-Jones, E. (2003) Anger and behavioral approach system. Personality and Individual Differences, 35, 995-1005.

Harmon-Jones, E., Sigelman, J. D., Bohlig, A., \& Harmon-Jones, C. (2003) Anger, coping, and frontal cortical activity: the effect of coping potential on anger-induced left frontal activity. Cognition and Emotion, 17, 1-24.

Hu, S., \& W WN, H. (2003) Imagined events with specific emotional valence produce specific patterns of facial EMG activity. Perceptual and Motor Skills, 97, 1091-1099.

IzARD, C. E. (1992) Basic emotions, relations among emotions, and emotion-cognition relations. Psychological Review, 99, 561-565.

KurNe, R. B. (2005) Principles and practice of structural equation modeling. (2nd ed.) New York: Guilford.

LaFrance, M., HBcht, M. A., \& Paluck, E. L. (2003) The contingent smile: a meta-analysis of sex differences in smiling. Psychological Bulletin, 129, 305-334.

Lang, P. J., Bradlex, M. M., \& CUTHBERT, B. (2005). International affective picture system (IAPS): instruction manual and affective ratings. Gainesville, FL: Univer. of Florida.

Lang, P. J., Greenwidid, M. K., Bradley, M. M., \& Hamm, A. O. (1993) Looking at pictures: affective, facial, visceral, and behavioral reactions. Psychopbysiology, 30, 261-273. 
Lazarus, R. S. (1991) Progress on a cognitive-motivational-relational theory of emotion. American Psychologist, 46, 819-834.

Leenen, I., van Mechelen, I, De Boeck, P, \& Rosenberg, S. (1999) INDCLAS; a three-way hierarchical classes model. Psychometrika, 64, 9-24.

Lerner, J. S., \& Keltner, D. (2001) Fear, anger, and risk. Journal of Personality and Social Psycbology, 8, 146-159.

Levenson, R. W., Carstensen, L. L., Friesen, W. V., \& EkMan, P. (1991) Emotion, physiology, and expression in old age. Psychology and Aging, 6, 28-35.

Levine, L. J. (1995) Young children's understanding of the causes of anger and sadness. Child Development, 66, 697-709.

Levine, L. J. (1996) The anatomy of disappointment: a naturalistic test of appraisal models of sadness, anger, and hope. Cognition and Emotion, 10, 337-359.

Lewis, M., Sullivan, M. W., Ramsey, D. S., \&Alessandri, S. M. (1992) Individual differences in anger and sad expressions during extinction: antecedents and consequences. Infant Behavior and Development, $15,443-452$.

Mikels, J. A., Fredrickson, B. L., Larkin, G. R., Lindberg, C. M., Maglio, S. J., \& ReuterLORENZ, P. A. (2005) Emotional category data on images from the International Affective Picture System. Behavior Research Methods, 37, 626-630.

Moll, J., de Oliveira-Souza, R., Eslinger, P. J., Bramati, I. E., Mourao-Miranda, J., Andreiuolo, P. A., \& PessoA, A. (2002) The neural correlates of moral sensitivity: a functional magnetic resonance imaging investigation of basic and moral emotions. Journal of Neuroscience, 22, 2730-2736.

Moll, J., de Oliveira-Souza, R., Moll, F. T., Ignacio, F. A., Bramati, I. E., Caparelli-Daquer, E. M., \& EsLINGer, P. J. (2005) The moral affiliations of disgust: a functional MRI study. Cognitive and Bebavioral Neurology, 18, 68-78.

Moltó, J., Montañés, S., Poy, R., Segarra, P, Pastor, M., \& Tokmo, P. (1999) Un nuevo método para el estudio experimental de las emociones: el international affective picture system (IAPS), adaptación española. Revista de Psicologia General y Aplicada, 52, 55-87.

MuRphy, S. T., \& ZAJONC, R. B. (1993) Affect, cognition, and awareness: affective priming with optimal and suboptimal stimulus exposures. Joumal of Personality and Social Psychology, 64, 723.739.

O'Donohue, W. T., \& Ferguson, K. E. (2003) Handbook of professional etbics for psychologists: issues, questions, and controversies. Thousand Oaks, CA: Sage.

Ortony, A., \& Turner, T. J. (1990) What's basic about basic emotions? Psychological Review, $97,315-331$.

Putcink, R (1980) A general psychoevolutionary theory of emotion. In R. Plutchik \& H. Kellerman (Eds.), Emotion: theory, research, and experience. Vol. 1. Theories of emotion. New York: Academic. Pp. 3-33.

RAmírez, J. M., \& ANDreu, J. M. (2006) Aggression, and some related psychological constructs (anger, hostility, and impulsivity); some comments from a research project. Neuroscience and Biobehavioral Review, 30, 276-291.

Ramírez, J. M., Fujihara, T., \& van Goozen, S. H. (2001) Cultural and gender differences in anger and aggression: a comparison between Japanese, Dutch and Spanish students. Journial of Social Psychology, 141, 119-121.

Rruddy, J. L., \& Wrlliams, A. E. (2005) Gender differences in pain: do enotions play a role? Gender Medicine, 2, 208-226.

RoBins, C. J., \& BLock, P. (1988) Personal vulnerability, life events, and depressive symptoms: a test of a specific interactional model. Journal of Personality and Social Psychology, 54, $847-852$

Smith, C. A, \& Lazarus, R. S. (1993) Appraisal components, core relational themes, and the emotions. Cognition and Emotion, 7, 233-269.

Spielberger, C. D., Jacobs, G. Russet.l, S. F., \& Crane, R. S. (1983) Assessment of anger: the State-Trait Anger Scale. In J. N. Butcher \& C. D. Spielberger (Eds.), Advances in personality assessment. Vol. 2. Fillsdale, NJ: Erlbaum. Pp. 159-187.

Spielberger, C. D., Rehreiser, E. C., \& Sydeman, S. J. (1995) Measuring the experience, expression, and control of anger. Issues in Comprebensive Pediatric Nursing, 18, 207-232.

Sternglanz, R. W., \& Dispaulo, B. M. (2004) Reading nonverbal cues to emotions: the advantages and liabilities of relationship closeness. Journal of Nonverbal Bebavior, 28, 245-266. 
Stroess: M. (2001) Gender differences in adjustment to bereavement: an empirical and theoretical review. Review of General Psychology, 5, 62-83.

SWAMM, R. C., HenRY, K. L., \& KELLY, K. (2006) Predictors of aggressive behaviors among rural middle school youth. The Journal of Primary Prevention, 27, 229-243.

TaKenara, T, \& Suzuki, N. (2001) Robustness of the two-dimensional structure of recognition of facial expression: evidence under different intensities of emotionality. Perceptual and Motor Skills, 93, 739-753.

UNDERWoOD, M. K. (2005) Observing anger and aggression among preadolescent girls and boys: ethical dilemmas and practical solutions. Ethics \& Bebavior, 15, 235-245.

VAN Mechelen, I., De Boeck, P, \& Rosinberg, S. (1995) The conjunctive model of hierarchical classes. Psychometrika, 60, 505-521.

Vila, J., Sánchez, M, Ramírez, I., Fernández, M., Cobos, P., Ronríguez, S., Muñoz, M. A., Tormo, P., Herrero, M., Segarra, P., Pastor, M. C., Montanés, S., Pay, R., \& Moltó, J. (2001) El sistema internacional de imágenes afectivas (IAPS): adaptación española, segunda parte. Revista de Psicología General y Aplicada, 54, 635-657.

Vonesh, E. F., \& ChInchilli, V. M. (1997) Linear and nonlinear models for the analysis of repeated measurements. New York: Marcel Dekker.

Weiner, B., Graham, S., \& Chandllir, C. (1982) Pity, anger, and guilt: an attributional analysis. Personality and Social Psychology Bulletin, 8, 226-232.

Accepted October 14, 2008 